\title{
Generalized Kalman Filter When Exogenous Variables Exist
}

\author{
Akira Yagi, Ritei Shibata \\ Department of Mathematics, \\ Keio University, 3-14-1 \\ Hiyoshi Kohoku, Yokohama, \\ 223-8522, Japan \\ yagi@math.keio.ac.jp, shibata@math.keio.ac.jp
}

\section{Abstract}

A generalized Kalman filter is proposed, which can be applied to any state space model with exogenous variables or inputs.

\section{State space model with exogenous variables}

We begin with a state space model,

$$
\begin{aligned}
x(t+1) & =F x(t)+L z(t)+\xi(t), \\
y(t) & =H x(t)+\eta(t) .
\end{aligned}
$$

Here $x(t)$ is an $n$-dimensional state vector and $z(t)$ is an $m$-dimensional vector of exogenous variables. The state $x(t)$ is updated to $x(t+1)$ with noise $\xi(t)$ as in (1). The output $y(t)$ is observed with noise $\eta(t)$ as in (2). The $F \in \boldsymbol{R}^{n \times n}, L \in \boldsymbol{R}^{m \times n}$ and $H \in \boldsymbol{R}^{n \times s}$ are matrices of the coefficients. We derive a generalized Kalman filter under the following assumptions.

$$
\begin{gathered}
E\left[\xi(s) \xi(t)^{\prime}\right]=\delta_{s t} Q, \quad E\left[\eta(s) \eta(t)^{\prime}\right]=\delta_{s t} R, \\
E\left[\xi(s) \eta(t)^{\prime}\right]=\delta_{s t} S, \\
E\left[\xi(t) x(0)^{\prime}\right]=E\left[\eta(t) x(0)^{\prime}\right]=0, \\
E\left[z(s) \xi(t)^{\prime}\right]=E\left[z(s) \eta(t)^{\prime}\right]=0, \\
E\left[x(0) z(s)^{\prime}\right]=0, \quad E\left[z(s) z(t)^{\prime}\right]=U(s, t), \\
Q \in \mathbb{R}^{n \times n}, \quad R \in \mathbb{R}^{s \times s}, \\
S \in \boldsymbol{R}^{n \times s}, \quad U(s, t) \in \mathbb{R}^{m \times m} .
\end{gathered}
$$

For simplicity we further assume that $x(0)$ is a random vector with $E[x(0)]=0$ and $E\left[x(0) x(0)^{\prime}\right]=\Sigma_{0}$ and that $z(t)$ is a random vector with $E[z(t)]=0$. We use the following notations,

$$
Y(t)=\left(\begin{array}{c}
y(0) \\
y(1) \\
\vdots \\
y(t)
\end{array}\right), \quad Z(t)=\left(\begin{array}{c}
z(0) \\
z(1) \\
\vdots \\
z(t)
\end{array}\right) .
$$

\author{
Takeshi Kato \\ School of Political Science and Economics, \\ Waseda University, 1-6-1 \\ Nishi-Waseda, Shinjuku, Tokyo \\ 169-8050, Japan \\ tkskato@mn.waseda.ac.jp
}

\section{Estimate of state vector}

We consider the case when $y(t)$ and $z(t)$ are both observable at time $t$. Let $\hat{y}(t \mid t-1)$ be the conditional expectation of $y(t)$ and $\hat{z}(t \mid t-1)$ be that of $z(t)$, given a $\sigma$-field $\mathcal{W}(t-1)$ generated by $w(0), w(1), \cdots, w(t-1)$, where

$$
w(t)=\left(\begin{array}{c}
y(t) \\
z(t)
\end{array}\right)
$$

By defining

$$
\hat{y}(t \mid t-1)=E[y(t) \mid \mathcal{W}(t-1)]
$$

and

$$
\hat{z}(t \mid t-1)=E[z(t) \mid \mathcal{W}(t-1)],
$$

we can write the conditional expectation of $w(t)$

$$
\hat{w}(t \mid t-1)=\left(\begin{array}{c}
\hat{y}(t \mid t-1) \\
\hat{z}(t \mid t-1)
\end{array}\right) .
$$

Denote the residual of $w(t)$ as

$$
e(t)=w(t)-\hat{w}(t \mid t-1),
$$

and the variance-covariance matrix as

$$
\Sigma(t)=E\left[e(t) e(t)^{\prime}\right]=\left(\begin{array}{ll}
\Sigma_{11}(t) & \Sigma_{12}(t) \\
\Sigma_{21}(t) & \Sigma_{22}(t)
\end{array}\right) .
$$

We also use the notation,

$$
e(t)=\left(\begin{array}{l}
e_{y}(t) \\
e_{z}(t)
\end{array}\right)=\left(\begin{array}{c}
y(t)-\hat{y}(t \mid t-1) \\
z(t)-\hat{z}(t \mid t-1)
\end{array}\right) .
$$

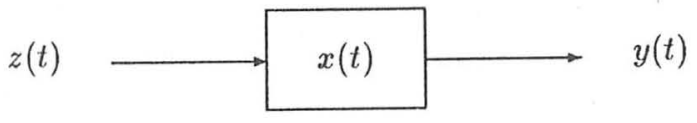

Fig. 1 : Linear system with exogenous variables $z(t)$ 
Theorem 1 Under the assumption that both $y(t)$ and $z(t)$ are Gaussian, a Kalman filter is given by the following formulas.

$$
\begin{aligned}
& \text { (i) } \quad \hat{x}(t+n \mid t)=F \hat{x}(t+n-1 \mid t) \\
& +L \hat{z}(t+n-1 \mid t) \quad(n>1) \\
& \text { (ii) } \hat{x}(t \mid t)=\hat{x}(t \mid t-1) \\
& +x x(t, t) H^{\prime} \Sigma(t)^{-1} e(t) \\
& \text { (iii) } \quad \hat{x}(t+1 \mid t)=F \hat{x}(t \mid t-1) \\
& +L z(t)+K(t) e(t) \\
& \text { (iv) } \quad e(t)=y(t)-H \hat{x}(t \mid t-1) \\
& \text { (v) } \quad \Sigma(t)=H x x(t, t) H^{\prime}+R \\
& \text { (vi) } \quad K(t)=\left(F x x(t, t) H^{\prime}+S\right) \Sigma(t)^{-1} \\
& \text { (vii) } \quad x x(t+1, t+1)=F x x(t, t) F^{\prime} \\
& +Q-K(t) \Sigma(t) K(t)^{\prime} \\
& \text { (viii) } \quad \hat{z}(t+n-1 \mid t) \\
& =a(t, t+n-1)^{\prime} U(t+1)^{-1} Z(t) \\
& \text { (ix) } \quad z z(t+1, t+1)=U(t+1, t+1) \\
& -a(t, t+1)^{\prime} U(t+1)^{-1} a(t, t+1) \\
& \text { (x) } \quad U(t+1)^{-1}=\left(\begin{array}{cc}
A(t) & B(t) \\
B(t)^{\prime} & z z(t, t)^{-1}
\end{array}\right) \text {, }
\end{aligned}
$$

where

$$
\begin{aligned}
& x x(t, t) \\
& \quad=E\left[(x(t)-\hat{x}(t \mid t-1))(x(t)-\hat{x}(t \mid t-1))^{\prime}\right] \\
& \begin{aligned}
x z(t, t) \\
\quad=E\left[(x(t)-\hat{x}(t \mid t-1))(z(t)-\hat{z}(t \mid t-1))^{\prime}\right]
\end{aligned} \\
& \begin{aligned}
z z(t, t) \\
\quad=E\left[(z(t)-\hat{z}(t \mid t-1))(z(t)-\hat{z}(t \mid t-1))^{\prime}\right] \\
\begin{aligned}
A(t) & =U(t)^{-1} \\
& +U(t)^{-1} a(t-1, t) z z(t, t)^{-1} a(t-1, t)^{\prime} U(t)^{-1}
\end{aligned} \\
B(t)=-U(t)^{-1} a(t-1, t) z z(t, t)^{-1} .
\end{aligned}
\end{aligned}
$$

Note that $\Sigma(t)$ and $e(t)$ are only a part of $\Sigma(t)$ or $e(t)$ defined previously. This is because we assume that $z(t)$ is observable together with $y(t)$ at time $t$ so that only the part related to $y(t)$ is necessary for updating the state $x(t)$.

\section{Proof.}

To prove the formulae in Theorem 1, we need the following Lemma 1. Although the proof of the Lemma 1 is placed in Appendix, we introduce the following notations.

$$
\begin{aligned}
& U(t)=\left(\begin{array}{ccc}
U(0,0) & \cdots & U(0, t-1) \\
\vdots & \ddots & \vdots \\
U(t-1,0) & \cdots & U(t-1, t-1)
\end{array}\right) \\
& U_{0}(t)=\left(\begin{array}{ccc}
U(0,0) & \cdots & U(0, t-1) \\
\vdots & \ddots & \vdots \\
U(t-2,0) & \cdots & U(t-2, t-1) \\
0 & \cdots & 0
\end{array}\right)
\end{aligned}
$$

$$
a(s, t)=\left(\begin{array}{c}
U(0, t) \\
\vdots \\
U(s, t)
\end{array}\right)
$$

$$
\begin{aligned}
& H(l)=\left(\begin{array}{ccc}
H & & 0 \\
& \ddots & \\
0 & & H
\end{array}\right), H(l) \in \mathbb{R}^{s l \times n l}, \\
& F(l)=\left(\begin{array}{cccc}
0 & & & 0 \\
I & \ddots & & \\
\vdots & \ddots & \ddots & \\
F^{l-2} & \ldots & I & 0
\end{array}\right),
\end{aligned}
$$$$
F(l) \in \mathbb{R}^{n l \times n l}
$$$$
F(p, q)=\left(\begin{array}{cccccc}
I & & & & & 0 \\
& \ddots & & & & \\
& & I & & & \\
& & & F & & \\
0 & & & & & \\
0 & & & \\
& & & & &
\end{array}\right) \text {, }
$$

$$
\begin{aligned}
& F(p, q) \in \boldsymbol{R}^{n p \times n p}, \\
& L(l)=\left(\begin{array}{ccc}
L & & 0 \\
& \ddots & \\
0 & & L
\end{array}\right), L(l) \in \boldsymbol{R}^{m l \times n l}, \\
& \Sigma_{0}(l)=\left(\begin{array}{ccc}
\Sigma_{0} & \cdots & \Sigma_{0} \\
\vdots & \ddots & \vdots \\
\Sigma_{0} & \cdots & \Sigma_{0}
\end{array}\right), \Sigma_{0}(l) \in \mathbb{R}^{n l \times n l} \\
& Q(q)=\left(\begin{array}{ccc}
Q & \cdots & Q \\
\vdots & \ddots & \vdots \\
Q & \cdots & Q
\end{array}\right), Q(q) \in \mathbb{R}^{n q \times n q}, \\
& Q(p, q)=\left(\begin{array}{cccccc}
0 & \cdots & 0 & 0 & \cdots & 0 \\
\vdots & \ddots & \vdots & \vdots & \ddots & \vdots \\
0 & \cdots & 0 & 0 & \cdots & 0 \\
0 & \cdots & 0 & & & \\
\vdots & \ddots & \vdots & & Q(q) \\
0 & \cdots & 0 & & &
\end{array}\right)
\end{aligned}
$$

$Q(p, q) \in \boldsymbol{R}^{n p \times n p}$,

$$
\begin{aligned}
& R(l)=\left(\begin{array}{cccc}
R & S^{\prime} H^{\prime} & \cdots & S^{\prime} F^{l-2} H^{\prime} \\
H S & R & \ddots & \vdots \\
\vdots & \ddots & \ddots & S^{\prime} H^{\prime} \\
H F^{l-2} & \cdots & H S & R
\end{array}\right), \\
& R(l) \in \mathbb{R}^{s l \times s l}
\end{aligned}
$$

Lemma 1 Assume that both $y(t)$ and $z(t)$ are Gaussian and $E\left[Y(t) Y(t)^{\prime}\right]$ is nonsingular when $L=0$, then we obtain the following formulae,

(i) $z(t)-\hat{z}(t \mid t-1)$ 
$=z(t)-a(t-1, t)^{\prime} U(t)^{-1} Z(t)$

(ii) $\quad x(t)-\hat{x}(t \mid t-1)=F^{t}(x(0)-\hat{x}(0 \mid t-1))$

$$
+\sum_{j=1}^{t} F^{j-1}(\xi(t-j)-\hat{\xi}(t-j \mid t-1))
$$

(iii) $\quad x(0)-\hat{x}(0 \mid t-1)$

$$
\begin{aligned}
& =x(0)-\left(\Sigma_{0} F^{\prime} H^{\prime}, \Sigma_{0} F^{\prime 2} H^{\prime}, \cdots, \Sigma_{0} F^{\prime t-1} H^{\prime}\right) \\
& \tilde{C}(t)^{-1} Y(t) \\
& +\left(\Sigma_{0} F^{\prime} H^{\prime}, \Sigma_{0} F^{\prime 2} H^{\prime}, \cdots, \Sigma_{0} F^{\prime-1} H^{\prime}\right) \\
& \tilde{C}(t)^{-1} H(t) F(t) L(t) U_{0}(t) U(t)^{-1} Z(t)
\end{aligned}
$$

(iv) $\xi(s)-\hat{\xi}(s \mid t-1)$

$$
\begin{aligned}
& =\xi(s)-\left(0, \cdots, 0, S, Q H^{\prime}, \cdots, Q F^{\prime t-1-s} H^{\prime}\right) \\
& \tilde{C}(t)^{-1} Y(t) \\
& +\left(0, \cdots, 0, S, Q H^{\prime}, \cdots, Q F^{\prime t-1-s} H^{\prime}\right) \tilde{C}(t)^{-1} \\
& H(t) F(t) L(t) U_{0}(t) U(t)^{-1} Z(t)
\end{aligned}
$$

where

$$
\begin{aligned}
\tilde{C}(t) & =H(t)\left\{F(t, t-1) \Sigma_{0}(t) F(t, t-1)^{\prime}\right. \\
& \left.+\sum_{s=0}^{t-2} F(t, s) Q(t, s+1) F(t, s)^{\prime}\right\} H(t)^{\prime}+R(t)
\end{aligned}
$$

and $s=0,1, \cdots, t-1$.

We now evaluate $x z(t, t)$. Since $x(t)-\hat{x}(t \mid t-1) \perp$ $\mathcal{W}(t-1)$ and $\hat{z}(t \mid t-1) \in \mathcal{W}(t-1)$

$$
x z(t, t)=E\left[(x(t)-\hat{x}(t \mid t-1)) z(t)^{\prime}\right] .
$$

Moreover since $x(t)-\hat{x}(t \mid t-1)$ is expressed by (ii), (iii) and (iv) in Lemma 1 and

$$
E\left[Y(t) z(t)^{\prime}\right]=H(t) F(t) L(t) U_{0}(t) U(t)^{-1} E\left[Z(t) z(t)^{\prime}\right],
$$

we obtain

$$
x z(t, t)=0 .
$$

For $e(t)$ since $\eta(t) \perp \mathcal{W}(t-1)$,

$$
e(t)=\left(\begin{array}{c}
y(t)-H \hat{x}(t \mid t-1) \\
z(t)-\hat{z}(t \mid t-1)
\end{array}\right) .
$$

About $\Sigma(t)$, we evaluate each block of the partitioned matrix,

$$
\Sigma(t)=\left(\begin{array}{ll}
E\left[e_{y}(t) e_{y}(t)^{\prime}\right] & E\left[e_{y}(t) e_{z}(t)^{\prime}\right] \\
E\left[e_{z}(t) e_{y}(t)^{\prime}\right] & E\left[e_{z}(t) e_{z}(t)^{\prime}\right]
\end{array}\right)
$$

Since $e_{y}(t)=H(x(t)-\hat{x}(t \mid t-1))+\eta(t)$ and $\eta(t) \perp\{x(t), \mathcal{W}(t-1)\}$, we obtain

$$
E\left[e_{y}(t) e_{y}(t)^{\prime}\right]=H x x(t, t) H^{\prime}+R .
$$

From the fact that $\eta(t) \perp\{z(t), \mathcal{W}(t-1)\}$, we have

$$
E\left[e_{y}(t) e_{z}(t)^{\prime}\right]
$$

Other blocks can be evaluated in the same manner. and we have

$$
\Sigma(t)=\left(\begin{array}{cc}
H x x(t, t) H^{\prime}+R & 0 \\
0 & z z(t, t)
\end{array}\right) .
$$

The formula (i) follows from

$$
\begin{aligned}
& \hat{x}(t+n \mid t)=F \hat{x}(t+n-1 \mid t) \\
& +L \hat{z}(t+n-1 \mid t)+\hat{\xi}(t+n-1 \mid t),
\end{aligned}
$$

since $\xi(t+n-1) \perp \mathcal{W}(t)$. To prove the equation (ii), we divide the space $\mathcal{W}(t)$ into the space $\mathcal{W}(t-1)$ and its tangent space $\mathcal{T}_{w}(t)$, which is spanned by the elements of $e(t)$. Then we can write

$$
\hat{x}(t \mid t)=\hat{x}(t \mid t-1)+K_{1}(t) e(t),
$$

for a $K_{1}(t) \in \boldsymbol{R}^{n \times(s+m)}$.

Then by solving

$$
0=E\left[(x(t)-\hat{x}(t \mid t)) e(t)^{\prime}\right]=\left(x x(t, t) H^{\prime}, x z(t, t)\right) \Sigma(t) .
$$

We have

$$
K_{1}(t)=\left(x x(t, t) H^{\prime}, 0\right) \Sigma(t)^{-1} .
$$

Moreover by partitioning $K_{1}(t)$ into $\left(K_{1}^{(1)}(t), K_{1}^{(2)}(t)\right)$, we have

$$
K_{1}^{(1)}(t)=x x(t, t) H^{\prime} \Sigma_{11}(t)^{-1}, \quad K_{1}^{(2)}(t)=0 .
$$

To show the equation (iii), we start from

$$
\hat{x}(t+1 \mid t)=F \hat{x}(t \mid t)+L z(t)+\hat{\xi}(t \mid t)
$$

and noting that

$$
\hat{\xi}(t \mid t)=E[\xi(t) \mid \mathcal{W}(t-1)]+E\left[\xi(t) \mid \mathcal{T}_{w}(t)\right],
$$

we see that $\hat{\xi}(t \mid t)$ can be written as

$$
\hat{\xi}(t \mid t)=K_{2}(t) e(t),
$$

since $\xi(t) \perp \mathcal{W}(t-1)$. Therefore,

$$
\begin{aligned}
\hat{x}(t+1 \mid t) & \\
= & F\left(\hat{x}(t \mid t-1)+\left(x x(t, t) H^{\prime}, x z(t, t)\right) \Sigma(t)^{-1} e(t)\right) \\
& +L z(t)+K_{2}(t) e(t) .
\end{aligned}
$$

Then we can rewrite the right hand side of the equation above and have

$$
\hat{x}(t+1 \mid t)=F \hat{x}(t \mid t-1)+L z(t)+K(t) e(t),
$$

by introducing a new $K(t)$. Moreover, since $x(t+1)-$ $\hat{x}(t+1 \mid t) \perp \mathcal{W}(t)$ and $e(t) \in \mathcal{W}(t)$,

$$
E\left[(x(t+1)-\hat{x}(t+1 \mid t)) e(t)^{\prime}\right]=0 .
$$

Thus

$$
K(t)=\left(F x x(t, t) H^{\prime}+S, 0\right) \Sigma(t)^{-1} .
$$


In a similar way, partitioning $K(t)$ into $\left(K^{(1)}(t)\right.$, $\left.K^{(2)}(t)\right)$, we have the desired result. We now evaluate $x x(t, t)$. Since

$$
\begin{aligned}
& x(t+1)-\hat{x}(t+1 \mid t) \\
& \quad=F(x(t)-\hat{x}(t \mid t-1))+\xi(t)-K(t) e(t),
\end{aligned}
$$

we obtain

$$
\begin{aligned}
x x( & +1, t+1)=F x x(t, t) F^{\prime} \\
& -F x x(t, t) H^{\prime} K(t)^{\prime}+Q \\
& -S K(t)^{\prime}-K(t) H x x(t) F^{\prime} \\
& -K(t) S^{\prime}+K(t) \Sigma(t) K(t)^{\prime} .
\end{aligned}
$$

We further employ the formula (vi), then

$$
\begin{aligned}
& F x x(t, t) H^{\prime}=K(t) \Sigma(t)-S \\
& H x x(t, t) F^{\prime}=\Sigma(t) K(t)^{\prime}-S^{\prime},
\end{aligned}
$$

and

$$
\begin{aligned}
x x(t+1, t+1)= & F x x(t, t) F^{\prime} \\
& +Q-K(t) \Sigma(t) K(t)^{\prime} .
\end{aligned}
$$

The formula (viii) easily follows from the equation (i) in Lemma 1. For $z z(t+1, t+1)$, since $z(t+1)-\hat{z}(t+1 \mid$ $t) \perp \mathcal{W}(t)$ and $\hat{z}(t+1 \mid t) \in \mathcal{W}(t)$ we have

$$
z z(t+1, t+1)=E\left[(z(t+1)-\hat{z}(t+1 \mid t)) z(t+1)^{\prime}\right],
$$

and

$$
\begin{aligned}
z z(t+1, t+1)= & U(t+1, t+1) \\
& -a(t, t+1)^{\prime} U(t+1)^{-1} a(t, t+1) \\
U(t+1)= & \left(\begin{array}{cc}
U(t) & a(t-1, t) \\
a(t-1, t)^{\prime} & U(t, t)
\end{array}\right) .
\end{aligned}
$$

The formula $(\mathrm{x})$ follows by a direct calculation the inverse matrix of $U(t+1)$. The proof of the theorem is now complete.

As an example of Theorem 1, consider the the case when no exogenous variable exists. We can rewrite the formulae in Theorem 1 to the following formulae,

$$
\begin{aligned}
\text { (i) } & \hat{x}(t+n \mid t)=F \hat{x}(t+n-1 \mid t) \quad(n>1) \\
\text { (ii) } & \hat{x}(t \mid t)=\hat{x}(t \mid t-1) \\
& \quad+x x(t, t) H^{\prime} \Sigma(t)^{-1}(t) \\
\text { (iii) } & \hat{x}(t+1 \mid t)=F \hat{x}(t \mid t-1) \\
& \quad+K(t) e(t) \\
\text { (iv) } & e(t)=y(t)-H \hat{x}(t \mid t-1) \\
\text { (v) } & \Sigma(t)=H x x(t, t) H^{\prime}+R \\
\text { (vi) } & K(t)=F x x(t, t) H^{\prime}+S \Sigma(t)^{-1}, \\
\text { (vii) } & x x(t+1, t+1)=F x x(t, t) F^{\prime} \\
& \quad+Q-K(t) \Sigma(t) K(t)^{\prime} .
\end{aligned}
$$

This is exactly the same as the Kalman filter which is well known.

We next consider the case when observation of $z(t)$ is lagged behind that of $y(t)$. That is, we redefine

$$
w(t)=\left(\begin{array}{c}
y(t) \\
z(t-1)
\end{array}\right) .
$$

We then obtain following theorem.

Theorem 2 Under the assumption that both $y(t)$ and $z(t)$ are Gaussian, we obtain following formulas,

(i) $\hat{x}(t+n \mid t)=F \hat{x}(t+n-1 \mid t)$

$$
\begin{aligned}
& +L \hat{z}(t+n-1 \mid t) \quad(n>1) \\
& t \mid t)=\hat{x}(t \mid t-1) \\
& +\left(x x(t, t) H^{\prime}, x z(t, t-1)\right) \Sigma(t)^{-1} e(t)
\end{aligned}
$$

(ii) $\quad \hat{x}(t \mid t)=\hat{x}(t \mid t-1)$$$
\hat{x}(t+1 \mid t)=F \hat{x}(t \mid t-1)
$$$$
+L \hat{z}(t \mid t)+K(t) e(t)
$$

$$
e(t)=\left(\begin{array}{c}
y(t)-H \hat{x}(t \mid t-1) \\
z(t-1)-\hat{z}(t-1 \mid t-1)
\end{array}\right)
$$$$
\Sigma(t)=\left(\begin{array}{cc}
H x x(t, t) H^{\prime}+R & H x z(t, t-1) \\
x z(t, t-1)^{\prime} H^{\prime} & z z(t-1, t-1)
\end{array}\right)
$$$$
K(t)=\left(F\left(x x(t, t) H^{\prime}, x z(t, t-1)\right)\right.
$$$$
\left.+L\left(x z(t, t)^{\prime} H^{\prime}, 0\right)+(S, 0)\right) \Sigma(t)^{-1}
$$$$
x x(t+1, t+1)=F x x(t, t) F^{\prime}+F x z(t, t) L^{\prime}
$$$$
+L x z(t, t)^{\prime} F^{\prime}+L z z(t, t) L^{\prime}
$$$$
+Q-K(t) \Sigma(t) K(t)^{\prime}
$$

(viii). $\quad \hat{z}(t+n-1 \mid t)$

$$
=a(t, t+n-1)^{\prime} U(t+1)^{-1} Z(t)
$$

$z z(t+1, t+1)=U(t+1, t+1)$

$$
-a(t, t+1)^{\prime} U(t+1)^{-1} a(t, t+1)
$$

$$
U(t+1)^{-1}=\left(\begin{array}{cc}
A(t) & B(t) \\
B(t)^{\prime} & z z(t, t)^{-1}
\end{array}\right)
$$

(xi) $\quad x z(t+1, t)=F x z(t, t)+L z z(t, t)$

(xii) $\quad x z(t, t)=L z z^{\prime}(t-1, t)-x z(t, t-1) B_{1}^{(t)^{\prime}}$

(xiii) $\quad z z^{\prime}(t, t+1)=U(t, t+1)$

$$
-a(t-1, t)^{\prime} U(t)^{-1} a(t-1, t+1),
$$

where

$$
\begin{aligned}
& x z(t, t) \\
& \quad=E[(x(t)-\hat{x}(t \mid t-1))(z(t)-\hat{z}(t \mid t))] \\
& z z(t, t) \\
& \quad=E\left[(z(t)-\hat{z}(t \mid t))(z(t)-\hat{z}(t \mid t))^{\prime}\right] \\
& z z^{\prime}(t, t+1) \\
& \quad=E\left[(z(t)-\hat{z}(t \mid t)) z(t+1)^{\prime}\right] \\
& \left(B_{t}^{(t)}, \cdots, B_{1}^{(t)}\right)=a(t-1, t)^{\prime} U(t)^{-1}
\end{aligned}
$$

The proof of Theorem 2 is omitted to save space, but it is similar to that of Theorem 1. Update scheme given in the Theorem 2 is illustrated in Fig. 2. 


$$
t-1 \text { step }
$$

$t$ step

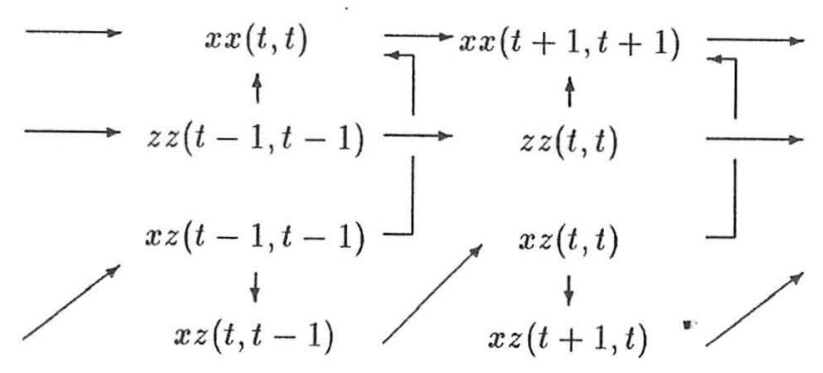

Fig. 2: Update scheme given in Theorem 2

\section{Appendix}

\subsection{Proof of Lemma 1}

Since $y(t)$ and $z(t)$ are Gaussian processes, we can write

$$
z(t)-\hat{z}(t \mid t-1)=z(t)-A^{(t)} Y(t)-B^{(t)} Z(t) .
$$

From

$$
E\left[(z(t)-\hat{z}(t \mid t-1)) Z(t)^{\prime}\right]=0
$$

and

$$
E\left[(z(t)-\hat{z}(t \mid t-1)) Y(t)^{\prime}\right]=0,
$$

we have the equations,

$$
\begin{gathered}
a(t-1, t)^{\prime}-A^{(t)} H(t) F(t) L(t) U_{0}(t) \\
-B^{(t)} U(t)=0 .
\end{gathered}
$$

and

$$
-A^{(t)} \tilde{C}(t)=0 .
$$

Here $\tilde{C}(t)=E\left[Y(t) Y(t)^{\prime}\right]$. The matrix $\tilde{C}(t)$ is of fullrank from the assumption, so that $A^{(t)}=0$ and,

$$
B^{(t)}=a(t-1, t)^{t} U(t)^{-1} .
$$

For $x(t)-\hat{x}(t \mid t-1)$, we have

$$
\begin{array}{r}
x(t)-\hat{x}(t \mid t-1)=F^{t}(x(0)-\hat{x}(0 \mid t-1)) \\
+\sum_{j=1}^{t} F^{j-1}(\xi(t-j)-\hat{\xi}(t-j \mid t-1)) .
\end{array}
$$

Since $y(t)$ and $z(t)$ are Gaussian processes, we can write

$$
\begin{aligned}
& x(0)-\hat{x}(0 \mid t-1)=x(0)-C^{(t)} Y(t)-D^{(t)} Z(t) \\
& \xi(s)-\hat{\xi}(s \mid t-1)=\xi(s)-M^{(t)} Y(t)-N^{(t)} Z(t), \\
& \text { for } s=0,1, \cdots, t-1 . \text { From } \\
& E\left[(x(0)-\hat{x}(0 \mid t-1)) Z(t)^{\prime}\right]=0, \\
& E\left[(x(0)-\hat{x}(0 \mid t-1)) Y(t)^{\prime}\right]=0, \\
& E\left[\xi(s)-\hat{\xi}(s \mid t-1) Z(t)^{\prime}\right]=0, \\
& E\left[\xi(s)-\hat{\xi}(s \mid t-1) Y(t)^{\prime}\right]=0 .
\end{aligned}
$$

we obtain

$$
\begin{array}{rc}
C^{(t)}= & \left(\Sigma_{0} H^{\prime}, \Sigma_{0} F^{\prime} H^{\prime}, \cdots, \Sigma_{0} F^{\prime t-1} H^{\prime}\right) \tilde{C}(t)^{-1}, \\
D^{(t)}= & -\left(\Sigma_{0} H^{\prime}, \Sigma_{0} F^{\prime} H^{\prime}, \cdots, \Sigma_{0} F^{\prime t-1} H^{\prime}\right) \tilde{C}(t)^{-1} \\
& H(t) F(t) L(t) U_{0}(t) U(t)^{-1} \\
M^{(t)}= & \left(0, \cdots, S, Q H^{\prime}, \cdots, Q F^{\prime t-1-s} H^{\prime}\right) \tilde{C}(t)^{-1} \\
N^{(t)}= & \left(0, \cdots, S, Q H^{\prime}, \cdots, Q F^{\prime t-1-s} H^{\prime}\right) \tilde{C}(t)^{-1} \\
& H(t) F(t) L(t) U_{0}(t) U(t)^{-1}
\end{array}
$$

\section{Concluding Remarks}

The Kalman filter we have derived is one of possible filters. For example, we can derive a different Kalman filter in which $Y(t)$ are used in preference to $Z(t)$. Wonham [2] derived a Kalman filter under the assumption that $Z(t)$ is measurable with respect to $Y(t)$. His filter is exactly the same as the filter given by (ii) to (ii) in Theorem 1. It is an interesting problem to find a Kalman filter when neither of $Z(t)$ nor $Y(t)$ is unobservable. We leave such interesting problems for future research.

\section{Reference}

[1] E.J.Hannan, Manfred Deistler, The statistical theory of linear systems, Wiley(1988)

[2] W.M.Wonham, Random differential equations in control theory, in Probabilistic Method in Applied Mathematics Vol.II, Ed. A.T.Bharucha-Reid, Academic Press(1970)

[3] T.Katayama, Oyo Kalman filter (Application of Kalman filter, in Japanese), Asakura(1983) 
